\title{
Síndrome Pfeiffer tipo 2
}

\author{
Pfeiffer syndrome type 2
}

\author{
Ever Domínguez-Morales, * Daniela del Carmen Aquino-Bonilla, ${ }^{\ddagger}$ Florencio Álvarez-Herbert, ${ }^{\S}$ \\ Humberto Martínez-García, ${ }^{\circledR}$ Clara Magdalena Martínez-Hernández" \\ * Departamento de Genética; ${ }^{\ddagger}$ Médico Residente de 4to año de Ginecología y Obstetricia; ${ }^{\S}$ División de Neonatología; ` División de \\ Neonatología; "| División de Investigación. Hospital Regional de Alta Especialidad de la Mujer, Villahermosa, Tabasco, México.
}

\begin{abstract}
RESUMEN
Introducción: El síndrome Pfeiffer es un trastorno autosómico dominante con incidencia de 1 en 100,000 recién nacidos, el cual se caracteriza por craneosinostosis y malformaciones en manos y pies. Caso clínico: Femenino de 26 días de edad, hija de primer embarazo de madre de 18 años y padre de 23 años; sin antecedente de consanguinidad o endogamia. Fue obtenida a término. Exploración física: cráneo en forma de trébol, fontanela anterior amplia, frente amplia, estrechez bitemporal, arcos supraorbitarios hipoplásicos y proptosis ocular bilateral. Extremidades superiores: pulgares anchos, clinodactilia de quinto dedo bilateral. Extremidades inferiores: primeros dedos anchos, clinodactilia de quinto dedo bilateral. En tomografía de cráneo: fusión de las suturas lambdoidea, sagital y coronal, asimetría de ventrículos, con disminución de la densidad del parénquima cerebral e hipoplasia cerebelar. La paciente falleció a los 35 días de vida. Conclusión: El espectro clínico y pronóstico de los pacientes con síndrome Pfeiffer es amplio y depende del tipo de mutación en genes FGFR1 y FGFR2, por lo cual requieren manejo multidisciplinario.
\end{abstract}

Palabras clave: Síndrome Pfeiffer, cráneo en trébol, craneosinostosis, genes FGFR1 y FGFR2.

\section{INTRODUCCIÓN}

El término craneosinostosis describe la fusión parcial o completa de las suturas craneales. Las craneosinostosis sindrómicas comprenden de $15-30 \%$ de los casos. ${ }^{1}$

\begin{abstract}
Introduction: Pfeiffer syndrome is an autosomal dominant disorder with an incidence of 1 in 100,000 newborns, which is characterized by craniosynostosis, besides hand and foot malformations. Clinical case: 26-day-old female, daughter of first pregnancy; 18-year-old mother and 23-year-old father, without consanguinity or inbreeding. It was obtained at term. Physical examination: cloverleaf skull, wide anterior fontanelle, wide forehead, bitemporal narrowing, hypoplastic supraorbital arches, and bilateral ocular proptosis. Upper extremities: broad thumbs, bilateral fifth finger clinodactyly. Lower extremities: broad first toes, bilateral fifth toe clinodactyly. Skull CT scan: fusion of the lambdoid, sagittal, and coronal sutures, ventricular asymmetry, with decreased density of the brain parenchyma, and cerebellar hypoplasia. At 35 days of life the patient died. Conclusion: Clinical and prognostic spectrum of patients with Pfeiffer syndrome is wide and depends on the type of mutation in FGFR1 and FGFR2 genes. These patients require multidisciplinary management.
\end{abstract}

Keywords: Pfeiffer syndrome, cloverleaf skull, craniosynostosis, FGFR1 and FGFR2 genes.

El síndrome Pfeiffer (SP) (acrocefalosindactilia tipo V, OMIM \#101600) es un trastorno autosómico dominante caracterizado por craneosinostosis, alteraciones en manos y pies. Es causado por mutaciones en FGFR1 (8p11.23) y FGFR2 (10q26.13), presenta penetrancia

Correspondencia: Ever Domínguez-Morales, E-mail: everdguez@msn.com

Citar como: Domínguez-Morales E, Aquino-Bonilla DC, Álvarez-Herbert F, Martínez-García H, Martínez-Hernández CM. Síndrome Pfeiffer tipo 2. Rev Mex Pediatr. 2021; 88(3): 112-115. https://dx.doi.org/10.35366/102192 
completa y heterogeneidad genética. La incidencia estimada es de 1 en 100,000-120,000 recién nacidos vivos y se han descrito tres fenotipos clínicos. ${ }^{2-5}$

Debido a los pocos reportes previos, presentamos el caso de una paciente con síndrome Pfeiffer tipo 2, a quien se le realizó el diagnóstico en el periodo neonatal.

\section{PRESENTACIÓN DEL CASO}

Femenino de 26 días de edad, hija del primer embarazo de una madre de 18 años de edad y padre de 23 años. Sin antecedente de consanguinidad, endogamia o de familiares con malformaciones (Figura 1).

La madre refiere adecuado control prenatal con ingesta de ácido fólico, hierro y calcio; ultrasonido obstétrico del tercer trimestre, el cual reportó alteración en la forma del cráneo del feto. Fue obtenida a término, por parto eutócico. La niña lloró y respiró al nacer.

Exploración física: peso $2,850 \mathrm{~g}$, talla $49 \mathrm{~cm}$. Cráneo en forma de trébol, fontanela anterior normotensa y amplia que llegaba al proceso nasal. Frente también amplia, no prominente; estrechez bitemporal y arcos supraorbitarios hipoplásicos. Puente nasal no deprimido, órbitas poco profundas, proptosis ocular bilateral; abultamiento en región mastoidea bilateral. Extremidades superiores: pulgares anchos, clinodactilia de quinto dedo bilateral. Extremidades inferiores: primeros dedos anchos, clinodactilia de quinto dedo bilateral (Figura 2).

Tomografía simple de cráneo: asimetría de los ventrículos, disminución en la densidad del parénquima cerebral, paquigiria e hipoplasia cerebelar. Mientras que en la tomografía de cráneo con reconstrucción tridimensional se observó fusión de la sutura lambdoidea, de la sutura sagital (parte posterior) y fusión de la sutura coronal (Figura 3).

La paciente falleció a los 35 días de vida, sin realizar estudios citogenéticos o moleculares.

\section{DISCUSIÓN}

El SP fue descrito por primera vez por Rudolph Pfeiffer en 1964, en ocho personas de tres generaciones de una misma familia. Los hallazgos descritos fueron: craneosinostosis bicoronal, hipoplasia media facial, alteraciones en los dedos de manos y pies, como sindactilia. ${ }^{6,7}$

En 1993 Cohen clasifica y describe tres fenotipos clínicos: tipo 1, considerado el síndrome clásico; tipo 2 , con cráneo en trébol, anquilosis de codos y sindactilia variable; y tipo 3 , similar al tipo 2 , pero sin cráneo en trébol. Con base en lo anterior, nuestra paciente

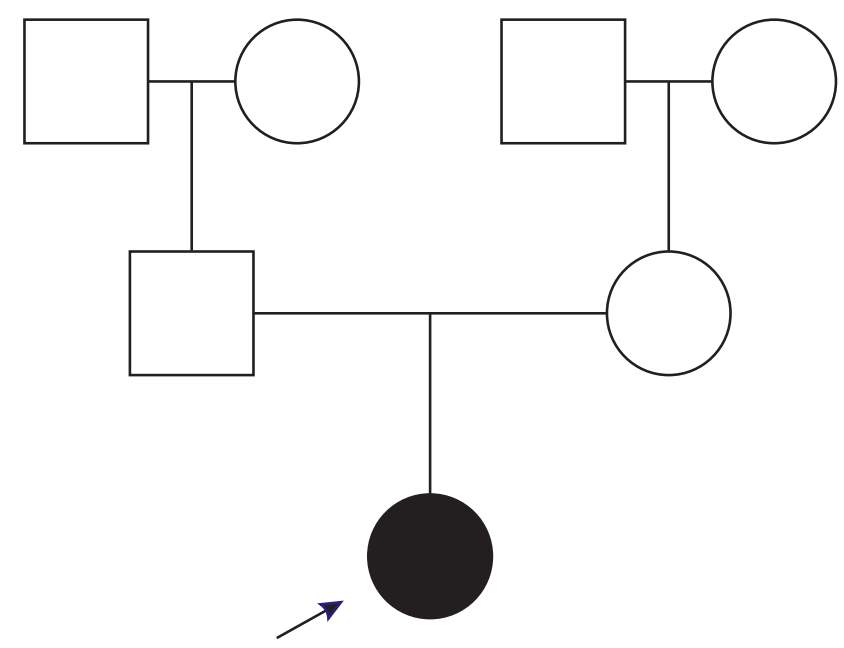

Figura 1: Árbol genealógico.

se puede clasificar en SP tipo 2. Los fenotipos 2 y 3 tienen pronóstico más pobre, debido al mayor número de alteraciones que se presentan. ${ }^{8,9}$

El SP es genéticamente heterogéneo; se han reportado alrededor de 25 mutaciones en FGFR1 y FGFR2, originando el $60 \%$ de los casos de este síndrome. La mayoría de los individuos afectados expresan mutación en FGFR2 y 5\% está asociado con mutación adicional de FGFR1. Las mutaciones en FGFR1 ocasionan un fenotipo leve (tipo 1). Los tipos 2 y 3 del síndrome son ocasionados por mutación en FGFR2, las mutaciones en este gen muestran expresividad clínica variable. ${ }^{10,11}$ La detección de la mutación correspondiente puede ayudar a determinar el riesgo de recurrencia.

La vía de señalización $F G F / F G F R$ desempeña una función esencial en numerosos procesos biológicos, entre los cuales se encuentra el desarrollo óseo y la homeostasis del control en la diferenciación de las células mesenquimales y neuroectodérmicas. Entre otras alteraciones, las mutaciones resultan en el cierre prematuro de las suturas. ${ }^{10}$

El cráneo en trébol es un signo frecuente en el SP tipo 2, causado por la fusión prematura de todas las suturas, excepto metópica y escamosa, produciendo abombamiento frontoparietal. Esta deformidad ósea se ha observado en las formas graves de los síndromes Crouzon, Apert, Carpenter, Saethre-Chotzen, así como en la displasia tanatofórica. ${ }^{12,13}$ En concordancia, en los hallazgos tomográficos de la paciente que se presenta se observó cráneo en trébol, por la fusión de las suturas lambdoidea, sagital y coronal.

El 40\% de los pacientes presentan hidrocefalia y, en algunos casos, malformación de Arnold-Chiari tipo 1. El 

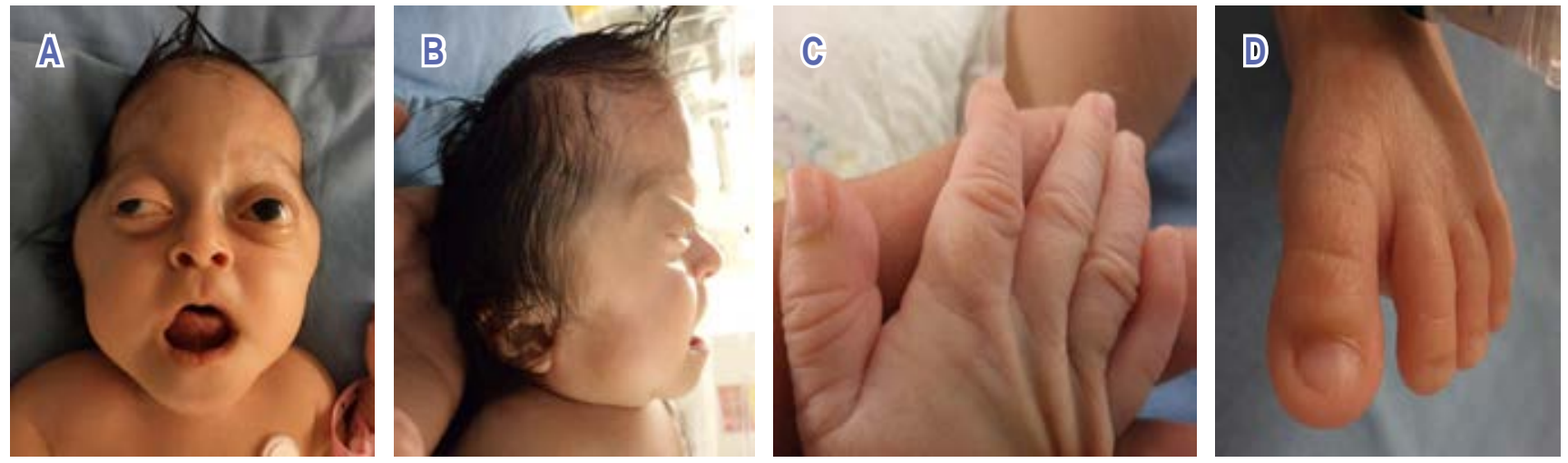

Figura 2: A) Cráneo en trébol, proptosis ocular; B) perfil facial plano, pabellones auriculares de implantación baja y displásicos; C) pulgar ancho y D) primer ortejo ancho (pie).
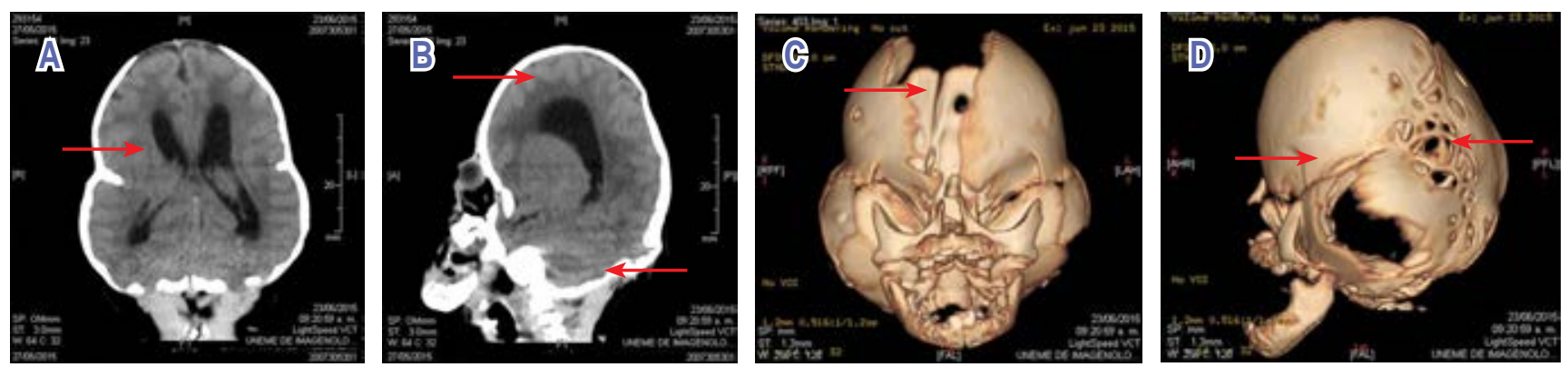

Figura 3: Tomografía de cráneo simple: A) asimetría de los ventrículos (flecha) y B) disminución de la densidad del parénquima cerebral e hipoplasia cerebelar (flechas). TAC de cráneo con reconstrucción tridimensional: C) fusión sutura sagital posterior (flecha) y D) sinostosis coronal y lambdoidea (flechas).

Tabla 1: Diagnóstico diferencial de pacientes que presentan fusión prematura de las suturas craneales.

\begin{tabular}{|c|c|c|c|c|c|c|}
\hline Sindrome & $\begin{array}{l}\text { Sutura craneal } \\
\text { afectada }\end{array}$ & $\begin{array}{l}\text { Alteraciones } \\
\text { en manos y pies }\end{array}$ & $\begin{array}{l}\text { Alteraciones } \\
\text { del SNC }\end{array}$ & Herencia & Gen & Locus \\
\hline Pfeiffer & Múltiples & $\begin{array}{l}\text { Pulgares y primeros ortejos } \\
\text { anchos, sindactilia parcial; } \\
\text { braquimesofalangia }\end{array}$ & $\begin{array}{l}\text { Hidrocefalia, } \\
\text { malformación de } \\
\text { Arnold-Chiari }\end{array}$ & $A D$ & FGFR1, FGFR2 & $8 p 11.23,10 q 26.13$ \\
\hline Crouzon & Múltiples & Sin alteraciones & Sin alteraciones & $A D$ & FGFR2 & 10q26.13 \\
\hline Apert & $\begin{array}{l}\text { Múltiples, } \\
\text { coronal }\end{array}$ & $\begin{array}{l}\text { Sindactilia cutánea y/o } \\
\text { ósea de manos y pies }\end{array}$ & $\begin{array}{c}\text { Hidrocefalia, } \\
\text { malformación de } \\
\text { Arnold-Chiari, agenesia } \\
\text { del cuerpo calloso }\end{array}$ & $A D$ & FGFR2 & $10 \mathrm{q} 26.13$ \\
\hline Saethre-Chotzen & Coronal & $\begin{array}{c}\text { Sindactilia, braquidactilia, } \\
\text { clinodactilia }\end{array}$ & $\begin{array}{l}\text { Retraso mental } \\
\text { leve a moderado }\end{array}$ & $A D$ & TWIST1 & $7 p 21.1$ \\
\hline Antley-Bixler & Múltiples & $\begin{array}{l}\text { Sinostosis radio-humeral, } \\
\text { aracnodactilia }\end{array}$ & $\begin{array}{l}\text { Hidrocefalia, } \\
\text { retraso mental }\end{array}$ & $A D, A R$ & FGFR2 & $10 \mathrm{q} 26.13$ \\
\hline
\end{tabular}

$\mathrm{SNC}=$ sistema nervioso central, $\mathrm{AD}$ = autosómico dominante; $\mathrm{AR}$ = autosómico recesivo. 
mal pronóstico de los tipos 2 y 3 , incluyendo la muerte temprana, es debido a complicaciones respiratorias o compromiso del sistema nervioso central. ${ }^{14}$ Esto último fue lo observado en la paciente descrita en este reporte.

El SP está dentro del grupo de síndromes que tienen como común denominador la craneosinostosis. En la Tabla 1 se presentan algunos datos que pueden ayudar al diagnóstico diferencial con los síndromes Crouzon, Apert, Saethre-Chotzen y Antley-Bixler.

El diagnóstico y manejo de los pacientes con $\mathrm{SF}$ debe ser por un equipo multidisciplinario, lo cual incluye identificar el pronóstico, manejo postnatal y, por supuesto, el asesoramiento genético a fin de la planificación genética-reproductiva de un siguiente embarazo.

\section{REFERENCIAS}

1. Ko JM. Genetic syndromes associated with craniosynostosis. $J$ Korean Neurosurg Soc. 2016; 59(3): 187-91. doi: 10.3340/ jkns.2016.59.3.187.

2. Jay S, Wiberg A, Swan M, Lester T, Williams LJ, Taylor IB et al. The fibroblast growth factor receptor 2 p.Ala172Phe mutation in Pfeiffer syndrome--history repeating itself. Am J Med Genet $A$. 2013; 161A(5): 1158-1163. doi: 10.1002/ajmg.a.35842.

3. Machado RA, Ferreira SB, Martins L, Ribero MM, Martelli DR, Coletta RD et al. A novel heterozygous mutation in FGFR2 gene causing Pfeiffer syndrome. Am J Med Genet A. 2017; 173(10): 2838-2843. doi: 10.1002/ajmg.a.38389.

4. Amiji I, Kalezi ZE, Abdulshakoor A, Tarimo JF, Leiya R, Zuechner A et al. Pfeiffer syndrome type 2; a case report of cranioorbitofaciostenosis with bilateral choanal atresia at Muhimbili National Hospital, Tanzania. Clin Case Rep. 2020; 8(9): 16131617. doi: $10.1002 /$ ccr3.2959.

5. Ben Hamouda H, Tlili Y, Ghanmi S, Soua H, Jerbi S, Souissi MM et al. Pfeiffer syndrome type II discovered perinatally: report of an observation and review of the literature. Diagn Interv Imaging. 2012; 93(10): 785-789. doi: 10.1016/j.diii.2012.06.002.
6. Lee MY, Jeon GW, Jung JM, Sin JB. A case of Pfeiffer syndrome with c833_834GC>TG (Cys278Leu) mutation in the FGFR2 gene. Korean J Pediatr. 2010; 53(7): 774-777. doi: 10.3345/ kjp.2010.53.7.774.

7. Júnior HM, de Aquino SN, Machado RA, Leão LL, Coletta RD, Burle-Aguiar MJ. Pfeiffer syndrome: clinical and genetic findings in five Brazilian families. Med Oral Patol Oral Cir Bucal. 2015; 20(1): e52-e58. doi: 10.4317/medoral.20032.

8. Huertas-Tacchino E, La Serna-Infantes J, Alvarado-Merino $\mathrm{R}$, Ingar-Pinedo J, Castillo-Urquiaga W, Zarate-Girao M et al. Síndrome de Pfeiffer tipo 2: diagnóstico prenatal. Reporte de caso y revisión de la literatura. Rev Peru Ginecol Obstet. 2019; 65(3): 361-366. doi: 10.31403/rpgo.v66i2196.

9. Torres-Canchala L, Castaño D, Silva N, Gómez AM, Victoria A, Pachajoa $\mathrm{H}$. Prenatal diagnosis of Pfeiffer syndrome patient with FGFR2 C.940-1G>C variant: a case report. Appl Clin Genet. 2020; 13: 147-150. doi: 10.2147/TACG.S251581.

10. Azoury SC, Reddy S, Shukla V, Deng CX. Fibroblast growth factor receptor 2 (FGFR2) mutation related syndromic craniosynostosis. Int J Biol Sci. 2017; 13(12): 1479-1488. doi: $10.7150 / \mathrm{ijbs} .22373$.

11. Rai R, Iwanaga J, Dupont G, Oskouian RJ, Loukas M, Oakes WJ et al. Pfeiffer type 2 syndrome: review with updates on its genetics and molecular biology. Child Nerv Syst. 2019; 35(1): 1451-1455. doi: 10.1007/s $00381-019-04082-7$.

12. Roldán-Arce J, Villarroel-Cortés C. Síndrome de Pfeiffer tipo 2. Informe de un caso y revisión de la literatura. Acta Pediatr Mex. 2013; 34(1): 43-47.

13. Flöttmann R, Knaus A, Zemojtel T, Robinson PN, Mundlos S, Horn D et al. FGFR2 mutation in a patient without typical features of Pfeiffer syndrome--The emerging role of combined NGS and phenotype based strategies. Eur J Med Genet. 2015; 58(8): 376380. doi: 10.1016/j.ejmg.2015.05.007.

14. Pfeifer CM. Kleeblattschädel in Pfeiffer syndrome type II. Radiol Case Rep. 2020; 15(5): 474-478. doi: 10.1016/j.radcr.2020.01.034.

Conflicto de intereses: los autores declaran que no tienen. 